\title{
Development of monitored tunable biodosimetry for fluence validation in an ultraviolet disinfection reactor
}

\author{
Mengkai Li ${ }^{\text {a }}$, Zhimin Qiang ${ }^{\mathrm{a}, *}$, Chen Wang ${ }^{\mathrm{a}}$, James R. Bolton ${ }^{\mathrm{b}}$, Junfeng Lian ${ }^{\mathrm{a}}$ \\ a State Key Laboratory of Environmental Aquatic Chemistry, Research Center for Eco-Environmental Sciences, Chinese Academy of Sciences, 18 Shuang-qing Road, \\ Beijing 100085, China \\ ${ }^{\mathrm{b}}$ Department of Civil and Environmental Engineering, University of Alberta, Edmonton, AB T6G 2W2, Canada
}

\section{A R T I C L E I N F O}

\section{Article history:}

Available online 26 January 2013

\section{Keywords:}

UV disinfection reactor

Fluence validation

Monitored tunable biodosimetry

Reduction equivalent fluence

Micro-fluorescent silica detector

\begin{abstract}
A B S T R A C T
The current biodosimetry, a primary fluence validation method for ultraviolet (UV) reactors, is confronting some serious challenges (e.g., the high cost and the lack of accurate monitoring of the lamp output), thus greatly limiting its practical application. In this study, a monitored tunable biodosimetry (MTB) was developed to validate the fluence of UV reactors with a lower cost but a higher accuracy. A novel microfluorescent silica detector (MFSD), used to monitor the real-time fluctuation of lamp output, was inserted into the gap between the lamp and the quartz sleeve. An adjustable ballast was utilized to tune the lamp output by adjusting the input voltage so as to export a desired reduction equivalent fluence. Fluence measurements were conducted on a commercial annular UV reactor with two challenge microorganisms (Bacillus subtilis spores and Escherichia coli). Results show that the MFSD could monitor well the lamp output during the validation process, and the viable number of $B$. subtilis spores could be greatly reduced by as much as 218 times. E. coli, a UV-sensitive microorganism usually inapplicable for fluence validation in the conventional biodosimetry, became applicable in the MTB process. Moreover, the dependence of fluence on water temperature could be readily determined. This MTB process cannot only increase the accuracy but also reduce the cost for UV fluence validation in comparison to the conventional biodosimetry. (c) 2013 Elsevier B.V. All rights reserved.
\end{abstract}

\section{Introduction}

In recent years, ultraviolet (UV) technology has been extensively applied to disinfect drinking water and municipal wastewater. Accurate validation of the delivered fluence (or UV dose) is crucial for the design of facility manufacturers, the operation and maintenance of users, and the site inspection of regulators as well. To date, both experimental and numerical methods have been developed to estimate the fluence delivery of UV reactors [1-5].

Biodosimetry, a primary experimental method applied to validate the fluence delivery of UV reactors with a nonpathogenic 'challenge' microorganism (e.g., MS-2 coliphage or Bacillus subtilis spores) over a range of operating conditions [6-9], has been commonly adopted as the standard method for UV fluence validation by many countries and organizations. In the test process, the inactivation ratio of the challenge microorganism, arising from UV exposure in a full-scale UV reactor, is used to back calculate the delivered fluence by comparing to a reference UV inactivation curve determined in laboratory with a quasi-collimated beam apparatus. The fluence (or UV dose) thus obtained is termed the reduction equivalent fluence (REF) (or the reduction equivalent

\footnotetext{
* Corresponding author. Tel.: +86 10 62849632; fax: +86 1062923541.

E-mail address: qiangz@rcees.ac.cn (Z. Qiang).
}

dose (RED)). The REF represents only an average fluence of the test UV reactor since microorganisms are actually exposed to a range of fluences depending on their numerous paths (or trajectories) along which they travel through the reactor $[10,11]$. The specific fluence received by each microorganism can be different because of the infinity of the potential travel paths of a microorganism and the fluence rate (FR) distribution in the UV reactor. Consequently, researchers have developed some experimental and numerical methods to determine the fluence distribution in a UV reactor [12-14].

An important advantage of the biodosimetry lies in that the test involves inactivation measurements of a waterborne microorganism in the field or at a test facility [15-18]. However, its practical application is confronting some serious challenges: (1) The realtime running status of a test UV reactor cannot be monitored accurately during the fluence validation process; in fact, the lamp often has a certain fluctuation in its output during the practical operation (e.g., as water temperature changes), despite being used after warming up; (2) The validation process requires cultivation of a large quantity of the challenge microorganism due to the high inactivation efficiency of UV lamps, which induces a high cost and workload; (3) Many challenge microorganisms have a narrow linear response range to the UV fluence, thus the inactivation saturation and minimal sensitivity threshold [14,15,19] frequently 
lead to inaccurate validation results; and (4) The biodosimetry only validates the fluence at one water temperature (due to its high cost and workload limitations), while water temperature usually fluctuates significantly during 1 day and exerts a strong impact on the lamp output.

In our previous work, a micro-fluorescent silica detector (MFSD) was developed for determination of FR distributions in UV reactors with different lamp configurations, such as a single-lamp reactor [20], a multiple-lamp reactor [21], and a reactor with various reactor walls [22]. Because the MFSD has distinguished features such as high stability, fast response, water resistance, and very small size, it can be easily inserted into the gap between the lamp and the associated quartz sleeve to monitor the real-time drift of lamp output during the practical operation of a UV reactor.

To overcome the above challenges faced by the conventional biodosimetry, this study aims to develop an improved method, namely 'monitored tunable biodosimetry (MTB)', to fast validate the fluence of a UV reactor with a lower cost but a higher accuracy. The MFSD, if installed in a UV reactor, can work as a lamp output detector (LOD) to measure continuously the real-time values of lamp output. Moreover, an adjustable ballast, which takes over the factory original one, can tune down stepwise the input voltage so as to reduce the lamp out to a desired level $\left(\operatorname{LOD}_{\mathrm{adj}}\right.$, monitored by the LOD) suitable for inactivation of the challenge microorganism. After conducting biodosimetry experiments at this reduced lamp output level, the REF ( $\left.R F_{a d j}\right)$ can be determined, which is afterwards converted to the original (or actual) REF ( $\mathrm{REF}_{\text {orig }}$ ) based on the ratio of the adjusted $\left(\mathrm{LOD}_{\mathrm{adj}}\right)$ to the original $\left(\mathrm{LOD}_{\text {orig }}\right)$ readings of the LOD. Tuning down the lamp output to a linear response fluence range will not only improve the measurement accuracy but also greatly reduce the required quantity of the challenge microorganism. Besides, through changing the water temperature and then recording the LOD reading, the REF values over a range of water temperatures can be readily determined. As a result, this new MTB provides a fast, accurate and cost-effective method for validation of UV fluence.

\section{Materials and methods}

\subsection{MTB validation procedures}

The primary problem of the conventional biodosimetry lies in its inability to monitor and control the lamp output during the validation process. However, the MTB can resolve this problem well with the combined assistance of an MFSD and an adjustable ballast. The detailed procedures for fluence validation with the MTB method are described as follows: (1) insert an MFSD (working as the LOD) into the gap between the UV lamp and the quartz sleeve to monitor and record the lamp output throughout the entire test process; (2) connect the factory original ballast to the lamp, run the test UV reactor at a required water temperature (controllable), and record the LOD reading ( $\left.\mathrm{LOD}_{\text {orig }}\right)$; $(3)$ replace the factory original ballast with an adjustable ballast, tune down stepwise the lamp output and record the LOD reading ( $\left.\operatorname{LOD}_{\mathrm{adj}}\right)$, and conduct the biodosimetry experiments at each reduced lamp output with a constant water temperature to obtain the corresponding REF ( $\left.R F_{a d j}\right)$ of the test UV reactor; and (4) calculate the original (or actual) REF $\left(\mathrm{REF}_{\text {orig }}\right)$ of the test reactor with equation:

$\mathrm{REF}_{\text {orig }}=\mathrm{REF}_{\mathrm{adj}} \times\left(\mathrm{LOD}_{\text {orig }} / \mathrm{LOD}_{\text {adj }}\right)$

Note that water temperature significantly affects the lamp output (and thus, the fluence delivery). To determine the water temperature dependence of the REF, the test UV reactor (connected to the original ballast) was allowed to run over a practical range of water temperatures, and the LOD reading at each water temperature $\left(\mathrm{LOD}_{\text {orig, } T}\right)$ was recorded. The corresponding $\mathrm{REF}\left(\mathrm{REF}_{\text {orig, }, T}\right)$ was thus calculated with equation:

$\mathrm{REF}_{\text {orig }, T}=\mathrm{REF}_{\text {adj }} \times\left(\mathrm{LOD}_{\text {orig }, T} / \mathrm{LOD}_{\text {adj }}\right)$

\subsection{Experimental set-up for the MTB process}

A schematic diagram of the experimental set-up for the MTB process is illustrated in Fig. 1. An annular commercial UV reactor (inner diameter $85 \mathrm{~mm} \times$ inner length $950 \mathrm{~mm}$ ) was utilized for experiments, which contained a low-pressure (LP) mercury lamp (GL, Xiashi Co., China; arc length $786 \mathrm{~mm}$, electric power $30 \mathrm{~W}$, UVC efficiency $28 \%$ ) centered inside a quartz sleeve (23 mm o.d.). The LP lamp could be connected to either a factory original ballast or an adjustable ballast. An MFSD was inserted into the gap between the UV lamp and the quartz sleeve (working as an LOD), whose signals were automatically recorded by a data acquisition switch unit (34971A, Agilent Co., USA). The UV-sensitive window of the LOD was located at about $180 \mathrm{~mm}$ from the left flange (see Fig. 1).

\section{3. $L O D_{\text {orig,T }}$ measurements}

To determine the $\mathrm{LOD}_{\text {orig, } T}$ values corresponding to various water temperatures under practical operating conditions, the test UV reactor (with the original ballast) was connected to a water recirculator that could precisely control the water temperature (see Fig. 1). The water was first cooled down to $5{ }^{\circ} \mathrm{C}$, and then the UV lamp was turned on to warm up for ca. 20 min to achieve a stable output when the $5{ }^{\circ} \mathrm{C}$ water was recirculated through the reactor. Afterwards, the cooling system was turned off and the lamp output was immediately monitored. Because the LP lamp continuously released heat into the water, the water temperature increased gradually from 5 to $38^{\circ} \mathrm{C}$ over a time period of $3 \mathrm{~h}$. The $\mathrm{LOD}_{\text {orig, },}$ and water temperature were simultaneously and continuously recorded by the data acquisition switch unit, which represented the operational status of the test UV reactor.

\subsection{Biodosimetry experiments}

B. subtilis spores (ATCC 6633) and E. coli (K-12) were individually used as the challenge microorganism, which were obtained from the Institute of Microorganisms, Chinese Academy of Sciences (Beijing, China), and propagated in our laboratory according to the reported methods $[23,24]$. The incubated challenge microorganisms were centrifuged $(10,000 \mathrm{rpm}, 10 \mathrm{~min})$ and washed for three times with a sterilized saline solution $(0.9 \%)$, and subsequently suspended in the sterilized saline solution $(500 \mathrm{~mL})$ as the stock solution.

For full-scale biodosimetry experiments, the factory original ballast was replaced by the adjustable ballast, and the inlet and

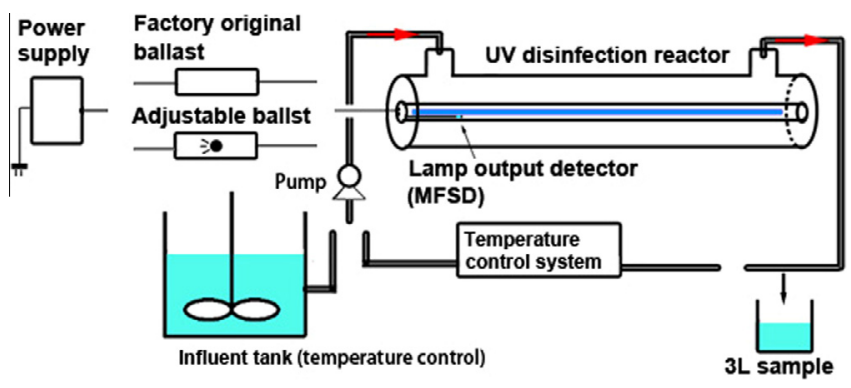

Fig. 1. Schematic diagram of the experimental set-up for the MTB process 
outlet of the test UV reactor were connected to a feed tank $(50 \mathrm{~L})$ and the sewer, respectively. A desired volume of the stock solution of the challenge microorganism was injected into the feed tank to reach approximately $10^{4}$ and $10^{6} \mathrm{CFU} / \mathrm{mL}$ for $B$. subtilis spores and $E$. coli, respectively. The UV transmittance (UVT) of the influent water was artificially adjusted by using instant coffee to $85 \%$, as verified with a Hach DR5000 UV-Vis spectrophotometer.

The biodosimetry experiments were performed to measure the microorganism inactivation ratios at various $\mathrm{LOD}_{\text {adj }}$ values through stepwise tuning down the adjustable ballast (i.e., the input voltage). The water temperature in the feed tank was controlled at $20{ }^{\circ} \mathrm{C}$ with an electric heating rod. Each measurement at a certain $\mathrm{LOD}_{\text {adj }}$ lasted for $1.25 \mathrm{~min}$ at a flow rate of $27.5 \mathrm{~L} / \mathrm{min}$.

Three liters of influent and effluent samples were collected, respectively. Afterwards, plate-count analysis was performed to determine the inactivation ratio of the challenge microorganism through a single 10 -fold serial dilution of each sample $(0.5 \mathrm{~mL})$ to $10^{-5}$. From each dilution, an aliquot of $1 \mathrm{~mL}$ sample was withdrawn, plated on three nutrient agar plates (beef extract $3.0 \mathrm{~g} / \mathrm{L}$, peptone $10.0 \mathrm{~g} / \mathrm{L}, \mathrm{NaCl} 5.0 \mathrm{~g} / \mathrm{L}$, agar $15.0 \mathrm{~g} / \mathrm{L}, \mathrm{pH}=7.2 \pm 0.2$ ), and incubated at $37^{\circ} \mathrm{C}$ for $24 \mathrm{~h}$. After incubation, the plate colonies were counted to determine the inactivation ratio. The detection limits for B. subtilis spores and E. coli were both $1 \mathrm{CFU} / \mathrm{mL}$.

Bench-scale experiments were performed to develop the fluence response curve of the challenge microorganism for the purpose of back calculating the fluence in the full-scale UV reactor. The challenge microorganisms (B. subtilis spores and $E$. coli) were respectively exposed to a range of known fluences supplied by an LP high output UV lamp (Light Sources Co., CA, USA; emission wavelength $253.7 \mathrm{~nm}$, electric power $105 \mathrm{~W}$, UVC efficiency 30\%) housed in a quasi-collimated beam apparatus. The experiments were conducted at an average incident fluence rate of $0.080 \mathrm{~mW} /$ $\mathrm{cm}^{2}$ [25], and the fluence was varied by changing the time of UV exposure to the challenge microorganism.

\section{Results and discussion}

\subsection{Total UVC output as a function of $L O D_{a d j}$}

In the MTB process, the LOD was fixed beside the UV lamp to monitor the lamp output. Because this detector had a small sensitive window (ca. $0.50 \mathrm{~mm}^{2}$ ), it could only receive the UV beams emitted by a certain part of the lamp (about $100 \mathrm{~mm}$ from the left electrode of the lamp). Therefore, it is important to determine the relationship between the total lamp output (in terms of UVC) and the $\mathrm{LOD}_{\text {adj }}$ reading.

The UV lamp (with the LOD) in the test reactor was pulled out and connected to the adjustable ballast for the total UVC output measurements. A radiometer (calibrated by the Chinese Metrology Institute, Beijing, China) was placed one meter away from the lamp in the air (see Fig. 2). When the input voltage was stepwise tuned down through the adjustable ballast, the radiometer and $\mathrm{LOD}_{\text {adj }}$ readings were recorded simultaneously. The UVC efficiency could then be calculated from the radiometer reading using the Keitz method [26].

Fig. 3 shows a good linear dependence of the irradiance on the $\mathrm{LOD}_{\text {adj }}$ over a broad range from 3 to $70 \mathrm{mV}\left(R^{2}=0.991\right)$. It demonstrates that the $\operatorname{LOD}_{\mathrm{adj}}$ reading could directly represent the total UVC output of the lamp in the MTB process.

\subsection{Monitoring and adjusting of the lamp output}

The test UV reactor was first connected to a water recirculator (water temperature controlled at $20^{\circ} \mathrm{C}$ ) to achieve a stable lamp output. After the lamp (connected to the factory original ballast)

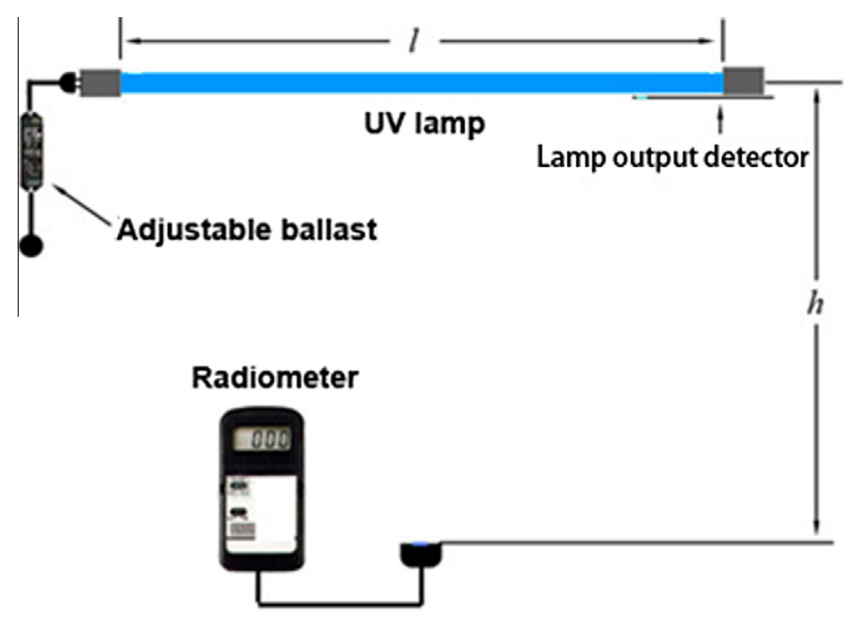

Fig. 2. Schematic diagram of the experimental set-up for measurement of the total UVC output as a function of $\mathrm{LOD}_{\mathrm{adj}}$.

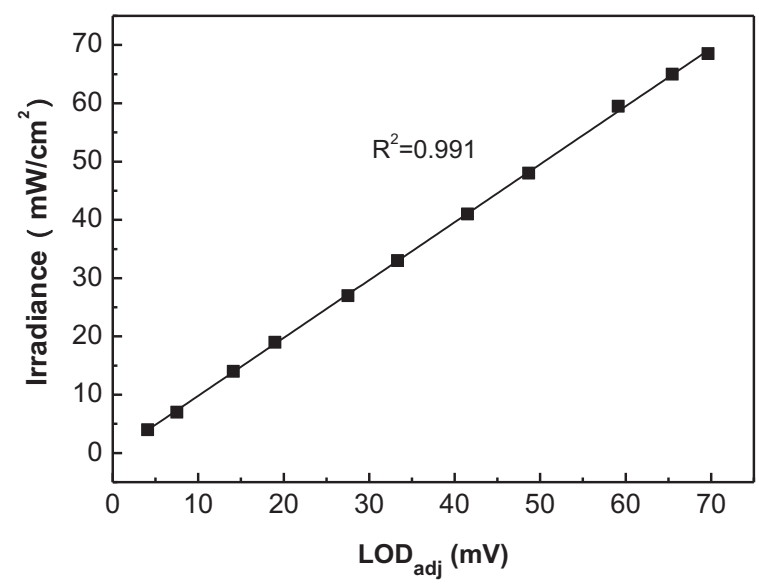

Fig. 3. Irradiance measured by a radiometer as a function of $L O D_{a d j}$.

was allowed to warm up enough at this controlled water temperature, the $\mathrm{LOD}_{\text {orig }}$ was measured as $65 \mathrm{mV}$.

The factory original ballast was then replaced by the adjustable ballast, and after disconnecting from the water recirculator, the UV reactor was connected to the influent tank containing the challenge microorganism solution with water temperature also controlled at $20^{\circ} \mathrm{C}$. Fig. 4 a shows the variation of the $\mathrm{LOD}_{\text {adj }}$ when stepwise tuning down the lamp output in the MTB process with $B$. subtilis spores as the challenge microorganism. There were totally eight stable lamp output stages (i.e., from B1 to B8). After the $\mathrm{LOD}_{\text {adj }}$ reached stable at each stage, the challenge microorganism solution was pumped to flow through the test reactor at a flow rate of $27.5 \mathrm{~L} / \mathrm{min}$. During the biodosimetry experiments, it was found that the $\mathrm{LOD}_{\mathrm{adj}}$ at each 'stable' stage had actually a slight fluctuation (less than 3\% difference between the maximum and average values) (Fig. $4 \mathrm{~b}$ ). Hence, the average $\mathrm{LOD}_{\mathrm{adj}}$ over the experimental period (i.e., $1.25 \mathrm{~min}$ ) was adopted to calculate the $\mathrm{REF}_{\text {orig }}$ from the above Eq. (1).

\subsection{Determination of $R E F_{\text {orig }}$}

The $\mathrm{REF}_{\mathrm{adj}}$ values were determined from the inactivation ratios $\left[\log \left(N_{o} / N\right)\right]$ of a $B$. subtilis spores suspension (UVT $=85 \%$, water temperature $=20^{\circ} \mathrm{C}$ ) at various stable lamp output stages, by com- 

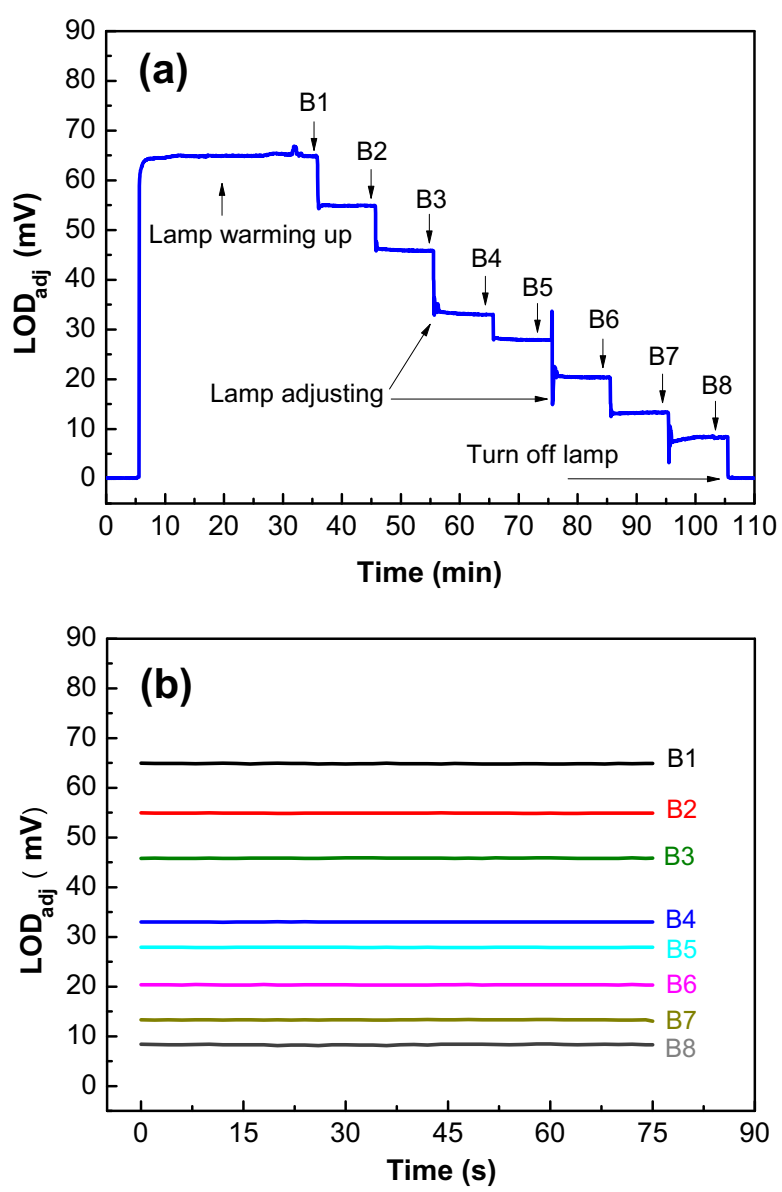

Fig. 4. Variation of $\mathrm{LOD}_{\mathrm{adj}}$ in the MTB process with B. subtilis spores: (a) during the entire lamp output tuning process; and (b) during each biodosimetry experiment.

Table 1

Determination of $\mathrm{REF}_{\text {orig }}$ at various lamp outputs using B. subtilis spores in the MTB process (water temperature $=20^{\circ} \mathrm{C}$ ).

\begin{tabular}{ccllll}
\hline No. $\begin{array}{l}\text { Ave. LOD adj } \\
(\mathrm{mV})\end{array}$ & $\begin{array}{l}\text { Relative } \\
\mathrm{UVC} \\
\text { output }(\%)\end{array}$ & $\log \left(N_{o} / N\right)$ & $\mathrm{REF}_{\text {adj }}\left(\mathrm{mJ} / \mathrm{cm}^{2}\right)$ & $\mathrm{REF}_{\text {orig }}\left(\mathrm{mJ} / \mathrm{cm}^{2}\right)$ \\
\hline B1 & 64.9 & 100 & 4.34 & 31.5 & \\
B2 & 54.9 & 85 & 3.95 & 28.5 & 31.5 \\
B3 & 45.8 & 71 & 3.51 & 25.2 & 33.7 \\
B4 & 33.0 & 51 & 2.36 & 16.5 & 32.5 \\
B5 & 27.9 & 43 & 2.00 & 13.8 & 32.2 \\
B6 & 20.4 & 31 & 1.05 & 6.7 & 21.3 \\
B7 & 13.3 & 20 & 0.65 & 3.7 & 18.1 \\
B8 & 8.3 & 13 & 0.28 & 0.9 & 7.0 \\
\hline
\end{tabular}

paring to the reference UV fluence response curve derived from the bench-scale experiments with a quasi-collimated beam apparatus (see Fig. S1, Supporting Information). Then, the corresponding $\mathrm{REF}_{\text {orig }}$ values could be readily calculated from Eq. (1) where the average $\mathrm{LOD}_{\text {adj }}$ was used, as shown in Table 1.

It was found that similar $\mathrm{REF}_{\text {orig }}$ values were obtained although the average $\mathrm{LOD}_{\text {adj }}$ decreased from 64.9 to $27.9 \mathrm{mV}$ (Table 1 ). It implies that by tuning the lamp output (i.e., $\mathrm{LOD}_{\text {adj }}$ ) down in the MTB process, a nearly same $\mathrm{REF}_{\text {orig }}$ could be acquired (mean $\pm \mathrm{SD}=33.1 \pm 1.7 \mathrm{~mJ} / \mathrm{cm}^{2}$ ). Meanwhile, the microorganism inactivation ratio decreased by $2.34 \mathrm{Log}$ as the $\mathrm{LOD}_{\text {adj }}$ was tuned down from 64.9 to $27.9 \mathrm{mV}$, which means that the viable number of the challenge microorganism could be reduced by as much as 218 times.
Table 2

Determination of $\mathrm{REF}_{\text {orig }}$ at various lamp outputs using E. coli in the MTB process (water temperature $=20^{\circ} \mathrm{C}$ ).

\begin{tabular}{llllll}
\hline No. & $\begin{array}{l}\text { Ave. } \\
\text { LOD adj } \\
(\mathrm{mV})\end{array}$ & $\begin{array}{l}\text { Relative } \\
\text { UVC } \\
\text { output }(\%)\end{array}$ & $\log \left(N_{\mathrm{o}} / \mathrm{N}\right)$ & $\mathrm{REF}_{\text {adj }}\left(\mathrm{mJ} / \mathrm{cm}^{2}\right)$ & $\mathrm{REF}_{\text {orig }}\left(\mathrm{mJ} / \mathrm{cm}^{2}\right)$ \\
\hline E1 & 29 & 45 & $\mathrm{~N} / \mathrm{A}^{\mathrm{a}}$ & $\mathrm{N} / \mathrm{A}$ & $\mathrm{N} / \mathrm{A}$ \\
E2 & 21.5 & 33 & $\mathrm{~N} / \mathrm{A}$ & $\mathrm{N} / \mathrm{A}$ & $\mathrm{N} / \mathrm{A}$ \\
E3 & 20 & 31 & 5.76 & 11.6 & 37.7 \\
E4 & 15 & 23 & 4.13 & 7.8 & 33.8 \\
E5 & 9.5 & 15 & 1.00 & 3.0 & 20.5 \\
\hline
\end{tabular}

a Not applicable.

It is noted that, when the average $\mathrm{LOD}_{\text {adj }}$ was further tuned down to a low range of $8.3-20.4 \mathrm{mV}$, much different $\mathrm{REF}_{\text {orig }}$ values were observed (i.e., $7.0-21.3 \mathrm{~mJ} / \mathrm{cm}^{2}$ ). As described above, the lamp irradiance showed a good linear dependence on the $\mathrm{LOD}_{\mathrm{adj}}$ in the $3-70 \mathrm{mV}$ range. Therefore, the smaller $\mathrm{REF}_{\text {orig }}$ values observed in the lower LOD $_{\text {adj }}$ range were most probably ascribed to the minimal sensitivity threshold of the challenge microorganism $[14,15,19]$. Many challenge microorganisms display a nonlinear response to an overly high or low fluence. Hence, by taking account of this effect, the lamp output should be adjusted to export a fluence range appropriate for inactivation of the challenge microorganism selected. In fact, the conventional biodosimetry is conducted from time to time under an inappropriate fluence because of the improper matching between the challenge microorganism and the practical fluence of a test UV reactor. However, in the MTB process, the biodosimetry accuracy can be obviously improved by adjusting the test reactor fluence into a linear response range for the challenge microorganism.

The MTB results with $E$. coli as the challenge microorganism are given in Table 2. Since E. coli is more sensitive to UV than B. subtilis spores, comparatively lower $\mathrm{LOD}_{\text {adj }}$ values were selected. Results indicate that at the average $\mathrm{LOD}_{\text {adj }}$ values of 29 and $21.5 \mathrm{mV}$, a complete inactivation of the spiked $E$. coli was found, and thus the logarithmic inactivate ratio $\left[\log \left(N_{o} / N\right)\right]$ could not be determined. When the $\mathrm{LOD}_{\mathrm{adj}}$ was tuned down to 20 and $15 \mathrm{mV}$, two similar $\mathrm{REF}_{\text {orig }}$ values were obtained (i.e., 37.7 and $33.8 \mathrm{~mJ} / \mathrm{cm}^{2}$ ). As the $\mathrm{LOD}_{\text {adj }}$ was further tuned to $9.5 \mathrm{mV}$, the $\mathrm{REF}_{\text {orig }}$ quickly dropped to $20.5 \mathrm{~mJ} / \mathrm{cm}^{2}$. The variation of $\mathrm{REF}_{\text {orig }}$ with the average $\mathrm{LOD}_{\text {adj }}$ for E. coli exhibited a similar trend to that for B. subtilis spores (see Table 1).

For the conventional biodosimetry, a number of UV-sensitive challenge microorganisms, such as E. coli and T1 phage, are not applicable at a high fluence (e.g., $40 \mathrm{~mJ} / \mathrm{cm}^{2}$ adopted for drinking water disinfection). However, in the MTB process, the biodosimetry can be performed with more challenge microorganism species at a monitored, tunable and appropriate fluence.

\subsection{Determination of $R E F_{\text {orig, }}$}

To determine the $\mathrm{REF}_{\text {orig, } T}$ values at various water temperatures, the test UV reactor was connected to the water recirculator (containing deionized water) and the lamp was connected to the factory original ballast with $\mathrm{LOD}_{\mathrm{adj}}$ adjusted to $27.9 \mathrm{mV}$ (B5, Table 1). The water temperature was varied in the practical range of $5-38^{\circ} \mathrm{C}$. Fig. 5 shows that the LOD $_{\text {orig. } T}$ increased first with an increasing water temperature till $32{ }^{\circ} \mathrm{C}$, and decreased afterwards. Through the above Eq. (2), a corresponding $\mathrm{REF}_{\text {orig, } T}$ curve could be derived as a function of water temperature (see Fig. 5). It is clearly seen that one outstanding advantage of the MTB is that it can determine the water temperature dependence of the $\mathrm{REF}_{\text {orig }}$ (i.e., fluence) much faster (measuring only the LOD $_{\text {adj }}$ values) and more easily (using only deionized water) than the conventional biodos- 


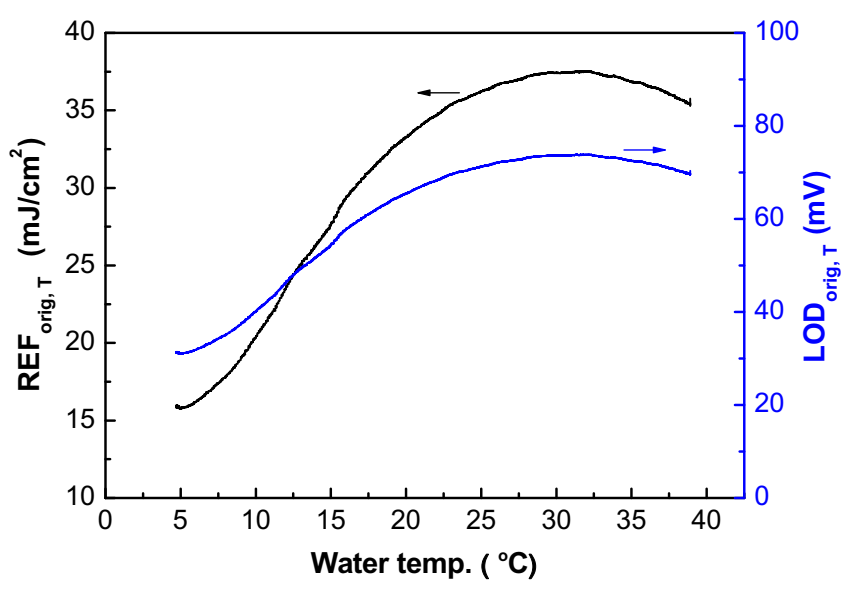

Fig. 5. Change of $\mathrm{REF}_{\mathrm{org}, T}$ and $\mathrm{LOD}_{\mathrm{org}, T}$ with water temperature.

imetry that has to measure the inactivation ratio of a challenge microorganism for each water temperature.

It is also noted that, to determine an appropriate $\mathrm{LOD}_{\text {adj }}$ range, the biodosimetry experiments were conducted at a series of $\mathrm{LOD}_{\text {adj }}$ values in this study. For practical application of the MTB process, one appropriate $\mathrm{LOD}_{\text {adj }}$ will actually be enough.

\section{Conclusions}

To push through the limitations of the conventional biodosimetry, this study developed an improved MTB method to fast validate the fluence of UV reactors with a lower cost but a higher accuracy. In brief, this new method has the following advantages:

- By using an MFSD (i.e., LOD) and an adjustable ballast, the lamp output can be monitored and adjusted throughout the entire test process, which significantly increases the measurement accuracy.

- The MTB process makes some UV-sensitive challenge microorganisms (e.g., E. coli) applicable for validation of a high fluence UV reactor.

- The viable number of the challenge microorganism can be greatly reduced.

- The MTB process can determine the water temperature dependence of the $\mathrm{REF}_{\text {orig }}$ (or practical fluence) much faster than the conventional biodosimetry.

\section{Acknowledgments}

This work was financially supported by the National Natural Science Foundation of China (51221892), the Ministry of Science and Technology of China (2012AA062606, 2012BAJ25B04), and the State Key Laboratory of Environment Aquatic Chemistry (12K01ESPCR). Dr. Bolton also thanks the Natural Sciences and Engineering Research Council of Canada for a Discovery Grant that partially supported this work.

\section{Appendix A. Supplementary material}

Supplementary data associated with this article can be found, in the online version, at http://dx.doi.org/10.1016/j.seppur.2013. 01.021 .

\section{References}

[1] C. Li, B.Q. Deng, C.N. Kim, A numerical prediction on the reduction of microorganisms with UV disinfection, AIChE J. 24 (2010) 1465-1473.

[2] B.A. Wols, J.A.M.H. Hofman, E.F. Beerendonk, W.S.J. Uijttewaal, J.C. van Dijk, A systematic approach for the design of UV reactors using computational fluid dynamics, AIChE J. 57 (2010) 193-207.

[3] D.A. Lyn, E.R. Blatchley III, Numerical computational fluid dynamics-based models of ultraviolet disinfection channels, J. Environ. Eng. ASCE 131 (2005) 838-849.

[4] D.A. Sozzi, F. Taghipour, UV reactor performance modeling by Eulerian and Lagrangian methods, Environ. Sci. Technol. 40 (2005) 1609-1615.

[5] S. Elyasi, F. Taghipour, Simulation of UV photoreactor for degradation of chemical contaminants: model development and evaluation, Environ. Sci. Technol. 44 (2010) 2056-2063.

[6] ÖNORM 2001, Austrian National Standard: ÖNORM M 5873-1 E, 2001, Plants for disinfection of water using ultraviolet radiation: requirements and testing, Part 1: Low pressure mercury lamp plants, Austrian Standards Institute, 2001. $<$ www.on-norm.at>.

[7] ÖNORM 2003, Austrian National Standard: ÖNORM M 5873-2 E. 2003, Plants for disinfection of water using ultraviolet radiation: requirements and testing, Part 2: Medium pressure mercury lamp plants, 2003. <www.on-norm.at>.

[8] DVGW 2006, UV devices for disinfection in drinking water supply, Part 1: Requirements on properties, function and operation, Part 2: Testing of properties, function and disinfection effectiveness, Part 3: Ports and sensors for the radiometric monitoring of UV disinfection devices - requirements, testing and calibration, 2006. <http://www.wvgw.de/index.php?id= 451\&submit_gesamtsuche=\&tx_indexedsearch\%5Bsword\%5D=W+294>.

[9] USEPA 2006, Ultraviolet disinfection guidance manual for the final long term 2 enhanced surface water treatment rule, 2006. <http://www.epa.gov/ safewater/disinfection/lt2/pdfs/guide_lt2_uvguidance.pdf $>$.

[10] J. Ducoste, D. Liu, K.G. Linden, Alternative approaches to modeling dose distribution and microbial in-activation in ultraviolet reactors: Lagrangian vs. Eulerian, J. Environ. Eng. ASCE 131 (2005) 1393-1403.

[11] K. Chiu, D.A. Lyn, P. Savoye, E.R. Blatchley III, Integrated UV disinfection model based on particle tracking, J. Environ. Eng. ASCE 125 (1999) 7-16.

[12] J. Ducoste, K.G. Linden, D. Rokjer, D. Liu, Assessment of reduction equivalent fluence bias using computational fluid dynamics, Environ. Eng. Sci. 22 (2005) $615-628$.

[13] E.R. Blatchley III, C.Y. Shen, O.K. Scheible, J.P. Robinson, L. Ragheb, D.E. Bergstrom, D. Rokjer, Validation of large-scale, monochromatic UV disinfection systems for drinking water using dyed microspheres, Water Res. 42 (2008) 677-688.

[14] Z. Bohrerova, G. Bohrer, S.M. Mohanraj, J. Ducoste, K.G. Linden, Experimental measurements of fluence distribution in a UV reactor using fluorescent microspheres, Environ. Sci. Technol. 39 (2005) 8925-8930.

[15] H. Mamane-Gravetz, K.G. Linden, A. Cabaj, R. Sommer, Spectral sensitivity of Bacillus subtilis spores and MS2 coliphage for validation testing of ultraviolet reactors for water disinfection, Environ. Sci. Technol. 39 (2005) 7845-7852.

[16] S. Jin, A. Mofidi, K.G. Linden, Polychromatic UV fluence measurements using chemical actinometry, biodosimetry, and mathematical techniques, J. Environ. Eng. ASCE 132 (2006) 831-841.

[17] A. Cabaj, R. Sommer, D. Schoenen, Biodosimetry: model calculations for UV water disinfection devices with regard to dose distributions, Water Res. 30 (1996) 1003-1009.

[18] M.T. Guo, H.Y. Hu, W.J. Liu, Preliminary investigation on safety of post-UV disinfection of wastewater: bio-stability in laboratory-scale simulated reuse water pipelines, Desalination 239 (2009) 22-28.

[19] W.L. Nicholson, B. Galeano, UV resistance of Bacillus anthracis spores revisited: validation of Bacillus subtilis spores as UV surrogates for spores of $B$. anthracis Sterne, Appl. Environ. Microbiol. 69 (2003) 1327-1330.

[20] M.K. Li, Z.M. Qiang, T.G. Li, J. Bolton, C.L. Liu, In situ measurement of UV fluence rate distribution by use of a microfluorescent silica detector, Environ. Sci. Technol. 45 (2011) 3034-3039.

[21] M.K. Li, Z.M. Qiang, J. Bolton, W.W. Ben, Impact of reflection on the fluence rate distribution in a UV reactor with various inner reactor walls as measured using a microfluorescent silica detector, Water Res. 46 (2012) 3505-3602.

[22] M.K. Li, Z.M. Qiang, J. Bolton, In situ detailed fluence rate distributions in a UV reactor with multiple low-pressure lamps: comparison of experimental and model results, Chem. Eng. J. 214 (2013) 55-62.

[23] M.T. Guo, J.J. Huang, H.Y. Hu, W.J. Liu, J. Yang, UV inactivation and characteristics after photoreactivation of Escherichia coli with plasmid: health safety concern about UV disinfection, Water Res. 46 (2012) 4031-4036.

[24] R. Sommer, A. Cabaj, D. Schoenen, J. Gebel, A. Kolch, A.H. Havelaar, F.M. Schets, Comparison of three laboratory devices for UV-inactivation of microorganisms, Water Sci. Technol. 31 (1995) 147-156.

[25] J. Bolton, K.G. Linden, Standardization of methods for fluence UV dose determination in bench-scale UV experiments, J. Environ. Eng. ASCE 131 (2003) 1393-1403.

[26] O. Lawal, B. Dussert, C. Howarth, K. Platzer, M. Sasges, J. Muller, E. Whitby, R. Stowe, V. Adam, D. Witham, S. Engel, P. Posy, A. van de Pol, Proposed method for measurement of the output of monochromatic (254 nm) low pressure UV lamps, IUVA News 10 (2008) 14-17. 


\section{Acronyms}

LOD: Lamp output detector

$L O D_{\text {adj: }}$ : LOD reading for a UV lamp connected to an adjustable ballast $(\mathrm{mV})$

$L O D_{\text {orig: }}$ LOD reading for a UV lamp connected to a factory original ballast ( $\mathrm{mV}$ )
MTB: Monitored tunable biodosimetry

$R E F_{\text {adj,T: }}$ REF value of a UV lamp connected to an adjustable ballast at a certain water temperature $\left(\mathrm{mJ} / \mathrm{cm}^{2}\right)$

$R E F_{\text {orig. } T \text { : }}$ REF value of a UV lamp connected to a factory original ballast at a certain water temperature $\left(\mathrm{mJ} / \mathrm{cm}^{2}\right)$ 